\title{
Global Positioning Strategies: A Comparison of Positioning Strategies Used in German and American Airline Magazine Advertisements: An Abstract
}

\author{
Charles Blankson, Selcuk Ertekin, and Cedric Lohse
}

\begin{abstract}
Due to increasing globalization and leveled down trade barriers between countries, companies can internationalize faster than ever before. Consequently, the creation of a global brand is becoming more and more important to businesses as they try to reach out to a global consumer culture. As the competition gets tougher in international domain, companies need to create positive connections in the target consumers' mind and have a clear positioning strategy. Carefully planned positioning strategies help establish brand equity and maintain consumers in the long term. In this study, global brand positioning is studied through advertisements in in-flight magazines for airlines from Germany and the United States. A consumer-based typology is used to identify and compare the positioning strategies used by the companies that advertise their products in these global magazines. The findings indicate that in-flight magazines from both of these domains utilize customer-derived positioning strategies in order to create consumer-based brand equity and strong consumer loyalty.
\end{abstract}

References Available Upon Request

\author{
C. Blankson \\ University of North Texas, Denton, TX, USA \\ e-mail: blanksoc@unt.edu \\ S. Ertekin $(\bowtie)$ \\ Brenau University, Gainesville, FL, USA \\ e-mail: sertekin@brenau.edu \\ C. Lohse \\ Missouri Western State University, Saint Joseph, MO, USA \\ e-mail: cedric.lohse91@gmail.com
}

